\title{
Virtual postoperative clinic: can we push virtual postoperative care further upstream?
}

\author{
Daniel Cornejo-Palma, ${ }^{1,2}$ David R Urbach ${ }^{1,3,4,5}$
}

${ }^{1}$ Institute for Health Policy, Management, and Evaluation, University of Toronto, Toronto, Canada

${ }^{2}$ Department of Surgery, University of Toronto, Toronto, Canada

${ }^{3}$ Department of Surgery, University of Toronto, Women's College Hospital, Toronto, Canada

${ }^{4}$ Women's College Hospital Institute for Health System Solutions and Virtual Care, Toronto, Canada

${ }^{5}$ Women's College Hospital Research Institute, Toronto, Canada

\section{Correspondence to}

Dr David R Urbach, Department of Surgery, University of Toronto, Women's College Hospital, Toronto, ON M5S 1B2, Canada; David.Urbach@wchospital.ca

Accepted 18 October 2018 Published Online First

31 October 2018

\section{Sinked}

- http://dx.doi.org/10.1136/ bmjqs-2018-008171

\section{Check for updates}

(C) Author(s) (or their employer(s)) 2019. No commercial re-use. See rights and permissions. Published by BMJ.

To cite: Cornejo-Palma $D_{\text {, }}$ Urbach DR. BMJ Qual Saf 2019;28:7-9.
Virtual care refers to remote healthcare interactions between patients and health professionals, predominantly using telecommunications networks. Virtual care interactions are a form of information exchange that guides care decisions. These interactions aim to enhance the patient experience and outcomes of care. Healthcare-related virtual care interactions can range from video clinic appointments to remote monitoring. ${ }^{1}$

The role of virtual care in surgery is rapidly evolving, and the nature of virtual interactions varies according to the phase of the surgical journey-spanning preoperative evaluation and assessment, preparation for surgery, intraoperative care and postoperative care.

The history of virtual surgical postoperative care actually goes back several decades. In a cohort of 536 patients with hip fracture published in 1990, telephone contact predicted return of function a year following surgery, likely due to improving patients' psychological function, reinforcing postoperative medication regimens and encouraging consistent participation in rehabilitation. ${ }^{2}$

Despite a rapidly evolving technological landscape, telephone contact with patients remains a cornerstone of remote interactions with patients after surgery. In this issue of BMJ Quality \& Safety, Healy et $a l^{3}$ report the results of a randomised controlled trial comparing a telephone-based virtual outpatient clinic with an actual outpatient clinic for the follow-up of general surgery patients 6-8 weeks after discharge from hospital. Of 107 subjects randomised to virtual follow-up, 98 (92\%) were successfully contacted by telephone, of which 10 $(10 \%)$ had postoperative issues and 3 of whom ultimately attended a conventional clinic for follow-up. Of 102 subjects randomised to the conventional outpatient clinic follow-up, 83 (81\%) attended the appointment, of which 16 (19\%) had postoperative concerns. Patients followed up by virtual care were more likely to be 'very satisfied' than conventional clinic follow-up (95\% vs 56\%); most patients in both groups expressed a preference for virtual follow-up in the future.

Virtual postoperative follow-up offers not just convenience-it may also improve care. The results reported by Healy and colleagues suggest that in-person follow-up in the clinic may result in inappropriate overuse of investigations. For example, 4 of 12 patients booked for follow-up endoscopy in the conventional care arm had no clear indication for a repeat procedure. Physicians might perceive greater pressure to recommend tests instead of offering reassurance for patients with mild postoperative symptoms, when facing an actual patient in clinic, already inconvenienced by a trip to the hospital outpatient clinic.

While patients clearly prefer virtual follow-up and there may also exist a salutary collateral reduction in inappropriate follow-up tests and procedures, we need to resist the temptation to overstate the potential benefits to the health system resulting from this model of care. Unless the virtual follow-up visits are largely automated-perhaps through automated telephone communications systems ${ }^{4}$ virtual follow-up visits will still require involvement of clinical care providers and their time will need to come from somewhere.

Whereas Healy et al emphasise the value of virtual follow-up after the completion of six to 8 weeks of surgical recovery, the greatest potential of postoperative virtual care likely lies upstream in the early postoperative phase, ${ }^{2}$ traditionally 
defined as the first 2 weeks after surgery. ${ }^{67}$ The term 'quality of recovery' has recently been used to describe the trajectory of a person's return to baseline function and quality of life following surgery, particularly with respect to limitations in self-care, pain, mood and mobility. ${ }^{6}$ A practical definition of good quality of recovery is function at $80 \%$ or more of baseline, without a drop in quality of life scores at postoperative day four. ${ }^{6}$ Poor quality of recovery impacts the shortterm experience of care and also has long-term implications. ${ }^{8}$ The ability to capture patient data remotely makes quality of recovery an appealing focus of postoperative virtual care. ${ }^{7}$

The focus on a quality of recovery measure of postoperative health is new. Historically, the quality of postoperative care was defined largely by the absence of major complications. ${ }^{6}$ Modern surgical and anaesthetic techniques have made major complications following surgery rare. Low major complications rates have made early discharge and day surgery popular with surgeons and patients. Early discharge has important potential benefits for health systems, saving up to $30 \%$ of the cost of hospitalisation for common surgical procedures. ${ }^{9}$ However, early discharge has potential risks. Leaving patients unsupervised in the early postoperative period exposes patients to preventable suffering. A 2-year cohort of 1118 day surgeries found nearly half of patients experienced poor quality recovery. ${ }^{6}$

Poor quality of recovery in the immediate postoperative period can cause long-term adverse outcomes. The risk of pain syndromes increases with increasing levels of uncontrolled acute postoperative pain ${ }^{8}$ following common procedures such as breast reconstruction, groin hernia repair and thoracotomy. ${ }^{810-12}$ Prolonged in-patient stays and early readmission after discharge are often a consequence of complicated early recovery phases and are associated with reduced long-term functional recovery, ${ }^{2}$ and a twofold increase in mean hospital costs. ${ }^{13}$ As early discharge becomes more common, increasing attention to early phase quality of recovery will become necessary to prevent adverse clinical and economic outcomes of poor quality of recovery.

The playing field of virtual surgical care is increasingly moving upstream into the early postoperative phase, with innovative smartphone apps focused on the measurement of quality of recovery, ${ }^{14-17}$ some of which have been evaluated in randomised controlled trials. ${ }^{18} 19$ Smartphone apps collect daily quality of recovery data to identify poor recovery for early intervention for procedures such as same day breast reconstruction $^{14}$ as well as a broad range of day surgery procedures. ${ }^{19}$ Telephone-based virtual clinics such as the one described by Healy et al offer convenience and enhanced experience of care, but miss the opportunity to improve quality of recovery earlier in the postoperative course.
What will the future of postoperative care mobile apps look like? Increasingly, virtual care will evolve from passive quality of recovery measurement to active real-time recovery. ${ }^{20}$ Real-time recovery uses quality of recovery assessments to implement early, aggressive course correction. ${ }^{7}$ For example, an ambulatory total knee replacement programme discharges patients home within 6 hours of surgery using a mobile app to track symptoms and biometric sensors to measure mobility, linking patients in real-time to the surgical team using 2-way video communication. ${ }^{21}$ Course correction of adverse symptoms can occur in real-time, in patients' homes. ${ }^{21}$

The real promise of virtual postoperative care is to move beyond remote identification of early complications and support real-time recovery that aims to identify and treat early phase complications aggressively. Enhancing the quality of recovery in the early postoperative period, particularly in the out-of-hospital settings which are increasingly common following ambulatory surgery, can improve long-term postoperative outcomes as well as enhance the acute postoperative patient experience. Virtual clinics will play an important role. But the biggest opportunities lie upstream, enhancing patients' quality of recovery in real time.

Funding The authors have not declared a specific grant for this research from any funding agency in the public, commercial or not-for-profit sectors.

Competing interests None declared.

Patient consent Not required.

Provenance and peer review Commissioned; internally peer reviewed.

\section{REFERENCES}

1 Shaw J, Jamieson T, Agarwal P, et al. Virtual care policy recommendations for patient-centred primary care: findings of a consensus policy dialogue using a nominal group technique. $J$ Telemed Telecare 2018;24:608-15.

2 Magaziner J, Simonsick EM, Kashner TM, et al. Predictors of functional recovery one year following hospital discharge for hip fracture: a prospective study. $J$ Gerontol 1990;45:M101-7.

3 Healy P, McCrone L, Tully R. Virtual outpatient clinic as an alternative to an actual clinic visit after surgical discharge: a randomised controlled trial. BMJ Qual Saf 2019;28:24-31.

4 Posadzki P, Mastellos N, Ryan R, et al. Automated telephone communication systems for preventive healthcare and management of long-term conditions. Cochrane Database Syst Rev 2016;12:CD009921.

5 Cleeland CS, Wang XS, Shi Q, et al. Automated symptom alerts reduce postoperative symptom severity after cancer surgery: a randomized controlled clinical trial. J Clin Oncol 2011;29:994-1000.

6 Stessel B, Fiddelers AA, Joosten EA, et al. Prevalence and predictors of quality of recovery at home after day surgery. Medicine 2015;94:e1553. 
7 Bowyer A, Royse C. The importance of postoperative quality of recovery: influences, assessment, and clinical and prognostic implications. Can J Anaesth 2016;63:176-83.

8 Kehlet H, Jensen TS, Woolf CJ. Persistent postsurgical pain: risk factors and prevention. Lancet 2006;367:1618-25.

9 Huang A, Ryu JJ, Dervin G. Cost savings of outpatient versus standard inpatient total knee arthroplasty. Can J Surg 2017;60:57-62.

10 Tasmuth T, Estlanderb AM, Kalso E. Effect of present pain and mood on the memory of past postoperative pain in women treated surgically for breast cancer. Pain 1996;68:343-7.

11 Callesen T, Bech K, Kehlet H. Prospective study of chronic pain after groin hernia repair. Br J Surg 1999;86:1528-31.

12 Katz J, Jackson M, Kavanagh BP, et al. Acute pain after thoracic surgery predicts long-term post-thoracotomy pain. Clin J Pain 1996;12:50-5.

13 Flynn DN, Speck RM, Mahmoud NN, et al. The impact of complications following open colectomy on hospital finances: a retrospective cohort study. Perioper Med 2014;3:1.

14 Semple JL, Armstrong KA. Mobile applications for postoperative monitoring after discharge. CMAJ 2017;189:E22-E24.

15 Nilsson U, Dahlberg K, Jaensson M. The swedish web version of the quality of recovery scale adapted for use in a mobile app: prospective psychometric evaluation study. JMIR Mhealth Uhealth 2017;5:e188.
16 Semple JL, Sharpe S, Murnaghan ML, et al. Using a mobile app for monitoring post-operative quality of recovery of patients at home: a feasibility study. JMIR Mhealth Uhealth 2015;3:e18.

17 Jaensson M, Dahlberg K, Eriksson M, et al. The development of the Recovery Assessments by Phone Points (RAPP): a mobile phone app for postoperative recovery monitoring and assessment. JMIR Mhealth Uhealth 2015;3:e86.

18 Armstrong KA, Coyte PC, Brown M, et al. Effect of home monitoring via mobile app on the number of in-person visits following ambulatory surgery: a randomized clinical trial. JAMA Surg 2017;152:622-7.

19 Dahlberg K, Philipsson A, Hagberg L, et al. Cost-effectiveness of a systematic e-assessed follow-up of postoperative recovery after day surgery: a multicentre randomized trial. Br J Anaesth 2017;119:1039-46.

20 Bowyer A, Royse CF. The future of postoperative quality of recovery assessment: multidimensional, dichotomous, and directed to individualize care to patients after surgery. Curr Opin Anaesthesiol 2016;29:683-90.

21 Favaro A. Home in six hours: Toronto hospital offers same-day joint replacement surgery [Internet]. CTV National News, 2018. Available from: https://www.ctvnews.ca/canada/home-insix-hours-toronto-hospital-offers-same-day-joint-replacementsurgery-1.3881534 [Accessed 11 May 2018]. 